\title{
Long-term Functional Outcomes for World Federation of Neurosurgical Societies Grade V Aneurysmal Subarachnoid Hemorrhage after Active Treatment
}

\author{
Kenichi ARIYADA, ${ }^{1}$ Tomoya OHIDA, ${ }^{1}$ Keita SHIBAHASHI, ${ }^{2}$ \\ Hidenori HODA, ${ }^{2}$ Kazuo HanAKawA, ${ }^{1}$ and Masahiko MURAO ${ }^{1}$ \\ ${ }^{1}$ Department of Neurosurgery, Tokyo Metropolitan Bokutoh Hospital, Tokyo, Japan \\ ${ }^{2}$ Tertiary Emergency Medical Center, Tokyo Metropolitan Bokutoh Hospital, Tokyo, Japan
}

\begin{abstract}
This study investigated the long-term outcomes of patients with World Federation of Neurosurgical Societies (WFNS) grade V aneurysmal subarachnoid hemorrhage (SAH) who underwent early aneurysm repair. We evaluated consecutive patients with WFNS grade V aneurysmal SAH from April 2010 to March 2015 who underwent aneurysm repair within $72 \mathrm{~h}$ after onset. We assessed the functional outcomes at discharge and 3 years after onset using the modified Rankin Scale (mRS). The primary outcome was defined as a favorable functional outcome $(\mathrm{mRS} \leq 2)$. We identified 145 patients with grade V SAH during the study period. Of these, 44 patients (19 males and 25 females; median age, 64 years; range, 24-79 years) met the inclusion criteria. For aneurysm repair, surgical clipping and coiling were performed in 40 $(90.9 \%)$ and $4(9.1 \%)$ patients, respectively. Although no patient had a favorable functional outcome at discharge, $11(25.0 \%)$ patients had a favorable functional outcome at the end of follow-up. The number of patients with a favorable outcome significantly increased during the first year $(P=0.012)$ and during the follow-up period $(P<\mathbf{0 . 0 0 1})$. Patients who underwent active rehabilitation had significant improvement. Our study showed that one-fourth of the patients who underwent early aneurysm repair with WFNS grade V SAH achieved a $\mathrm{mRS}$ score of $\leq 2$ over a 3 -year period. It might be important to consider age and rehabilitation for better clinical outcomes. Larger studies are required to adequately assess the long-term functional outcomes and other multi-faceted prognoses.
\end{abstract}

Keywords: aneurysmal subarachnoid hemorrhage, early aneurysm repair, modified Rankin scale, World Federation of Neurosurgical Societies Grade V

\section{Introduction}

In patients with aneurysmal subarachnoid hemorrhage (SAH), the severity of their neurological condition at admission is the strongest prognostic indicator. ${ }^{1)}$ The most severe grade of SAH, corresponding to World Federation of Neurosurgical Societies (WFNS) grade $\mathrm{V}$, accounts for approximately $20 \%$ of all hospitalized $\mathrm{SAH}$ cases, ${ }^{2}$ and is associated with a poor functional outcome. Because majority of the patients with $\mathrm{SAH}$ experience initial damage, intracranial hypertension, cerebral infarctions, rebleeding in the acute stage, ${ }^{3,4)}$ and medical complications, those with grade V SAH are often excluded from active treatment. ${ }^{5)}$

Received February 18, 2020; Accepted April 27, 2020

Copyright $@ 2020$ by The Japan Neurosurgical Society This work is licensed under a Creative Commons AttributionNonCommercial-NoDerivatives International License.
Some studies have reported the benefits of intensive care, ${ }^{6)}$ early aneurysm repair, ${ }^{7)}$ and emergency surgical intervention (particularly in patients with hematomas ${ }^{8)}$ in the treatment of most severe cases of SAH. However, because existing data on the longterm functional outcomes of such patients are scarce, the optimal treatment strategy for patients with grade V SAH remains unclear. Thus, further study is required to address this gap in knowledge.

In the present study, we investigated the longterm functional outcomes of patients who underwent aggressive treatment for WFNS grade V SAH.

\section{Materials and Methods}

This retrospective cohort study was approved by the Ethics committee and the Institutional Review Board of the Tokyo Metropolitan Bokutoh Hospital, Japan (approval number: 31-052), and some data were anonymized before analysis. The need for informed 


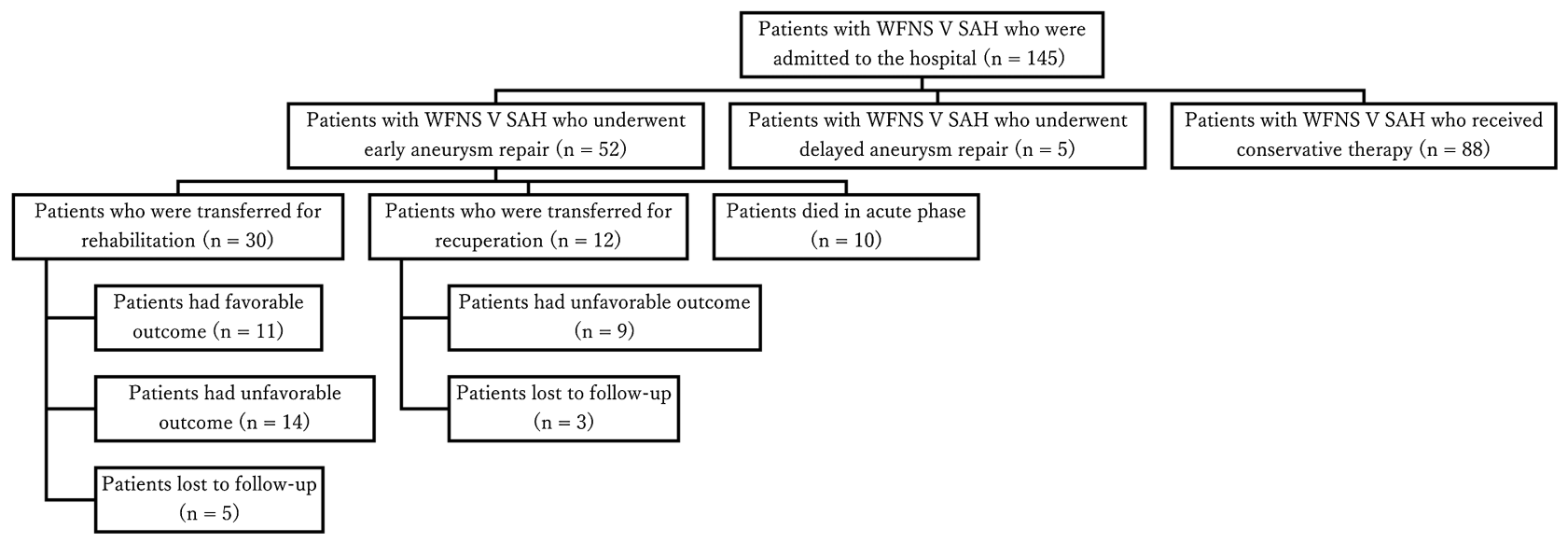

Fig. 1 Study population and flowchart of 145 patients with WFNS grade V subarachnoid hemorrhage. The outcomes are shown in relation to different facilities after transferal from an acute care hospital. WFNS: world federation of neurosurgical societies.

consent was waived because of the retrospective study design. With regard to the use of data obtained by telephonic interview, oral patient consent was obtained over the telephone. This study was performed according to the principles of the Declaration of Helsinki. We investigated patients who were admitted to our hospital with WFNS grade V SAH between April 2010 and March 2015 (Fig. 1). The WFNS grading system is based on the consciousness level (Glasgow Coma Scale [GCS]) and neurological symptoms (motor deficit). ${ }^{9}$ Inclusion criteria were as follows: (1) non-traumatic SAH of WFNS grade V and (2) early aneurysm repair within $72 \mathrm{~h}$ after onset. Patients who received early surgical or endovascular therapy were excluded if there was a failure to follow-up within the observation period.

In our 24-bed intensive care unit, medical care is provided by full-time staff members, including neurosurgeons and intensivists. The diagnosis of $\mathrm{SAH}$ was based on the results of computed tomography (CT). WFNS grade was determined at the time of hospitalization by the attending surgeon who decided the treatment strategy. ${ }^{6)}$ Unless the futility of active treatment was certain, early surgical repair of the aneurysm was considered. Active treatment was declared futile if the patient was resuscitated after cardiac arrest, had bilateral dilatation of the pupils $(\geq 5 \mathrm{~mm})$, or had signs of brainstem functional disorders, as these signs indicated that the possibility of recovery was expected to be very low. ${ }^{10)}$

Treatments for the prevention of cerebrovascular spasm included external ventricular drainage (EVD) and the administration of fasudil (30 mg every 8 hours, after aneurysm repair starting from the 14th day after onset), ozagrel (80 mg per day, as a constant infusion throughout the same period as abovementioned), and nicardipine (24-240 mg per day, depending on the blood pressure, as a constant infusion during the same period as above). All patients underwent rehabilitation in the acute stage if conditions permitted. After discharge, patients were moved to a facility with or without rehabilitation services, based on the anticipated need for further active rehabilitation.

The collected patient data included age, sex, history (hypertension, diabetes mellitus, and dyslipidemia), length of hospital stay, clinical presentation, and radiological findings (GCS score, ventricular or cerebral clot, diameter (divided into $<10 \mathrm{~mm}$ or $\geq 10 \mathrm{~mm})^{11)}$ and location of aneurysms, initial systolic blood pressure, time to aneurysmal repair, and modality of aneurysmal repair. Acute hydrocephalus was defined as an adjusted relative bicaudate index above $1^{12)}$ on initial CT images. Ischemia by cerebrovascular spasm during the acute stage is defined as finding from the study of images; vasospasm and brain infarction by CT or magnetic resonance imaging examinations after neurological changes; or as periodic tests.

Patients' outcomes were assessed using the modified Rankin Scale (mRS) at hospital discharge, and with interviews performed at an outpatient clinic (or over telephone) by the authors, 1-3 years after the hemorrhage. The mRS measures functional independence and has been frequently used for the evaluation of stroke-related handicap in many trials. ${ }^{13)}$ The long-term outcome was classified as favorable (mRS $\leq 2)$ or unfavorable $(\mathrm{mRS}>2$ ).

We evaluated differences between patients with favorable and unfavorable outcomes using the $\chi^{2}$ test or Fisher's exact test for clinical findings, CochranArmitage test for categorical data, and Mann-Whitney $\mathrm{U}$ test for continuous data. In addition, the receiver 


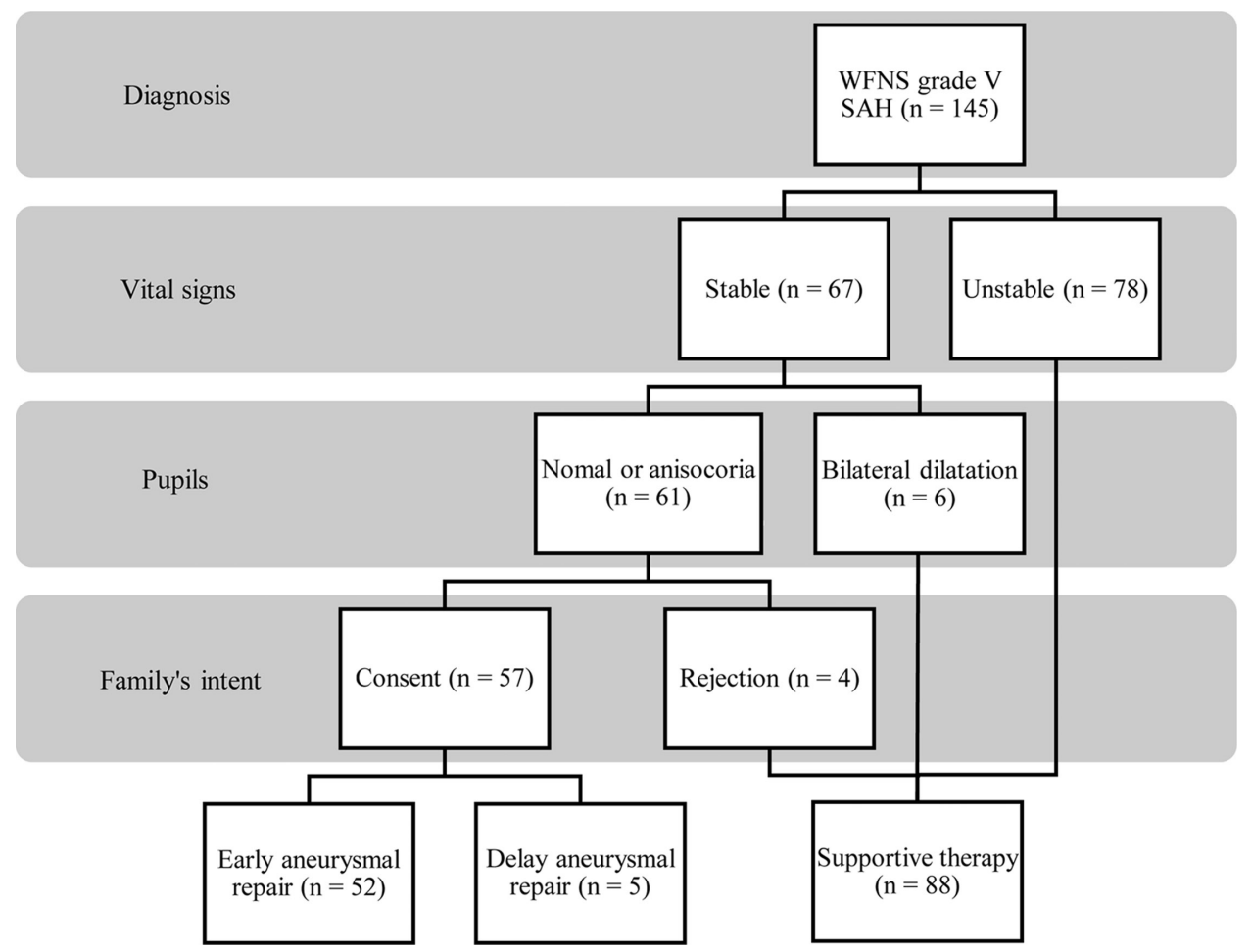

Fig. 2 Flowchart of the indications and strategies for 145 patients with WFNS grade V subarachnoid hemorrhage. WFNS: world federation of neurosurgical societies.

operating characteristic (ROC) curve was analyzed for evaluating age and long-term outcomes, and the following cutoff was selected on the basis of the highest sensitivity and specificity values generated from the ROC curve analysis. All $P$ values were two-sided and $P$ values of 0.05 or less were considered statistically significant. All statistical analyses were performed with EZR (Saitama Medical Center, Jichi Medical University, Saitama, Japan), which is a graphical user interface for R (The R Foundation for Statistical Computing, Vienna, Austria). ${ }^{14)}$

\section{Results}

In all, 145 patients were diagnosed with WFNS grade V SAH during the study period. Of these, 52 $(35.9 \%)$ patients underwent early aneurysm repair based on the inclusion criteria, $5(3.4 \%)$ patients underwent delayed aneurysm repair, and 88 (60.7\%) patients underwent conservative therapy (Fig. 1). Among patients who underwent delayed surgery, four patients gradually recovered after undergoing an emergency EVD, and the neurosurgical team decided to perform a delayed aneurysm repair (range, 5-26 days after admission); three patients underwent endovascular treatment; and one patient underwent clipping. Only one patient had an unclear rupture point based on the initial digital subtraction angiography (DSA). After the third DSA, the neurosurgical team recognized a vertebral aneurysm and performed a coil embolization 8 days after admission. Figure 2 shows the detailed reasons for patients who did not undergo aggressive therapy. The most common reason was unstable vital signs at primary care $(\mathrm{n}=78 ; 88.6 \%)$, including resuscitation after cardiac arrest $(\mathrm{n}=23 ; 2.1 \%)$. Other reasons were bilateral pupil dilatation $(\mathrm{n}=6 ; 6.8 \%)$ and disagreement of the patients' families to aggressive treatment ( $\mathrm{n}=4 ; 4.5 \%$ ), based on the patients' desires, their activity of daily living, and medical history. All such patients died during the acute stage.

Based on the exclusion criterion, 8 of 52 cases with early aneurysm repair were excluded from the analysis. Table 1 shows the baseline characteristics of the patients identified as being eligible for this study. This study population consisted of 19 males and 25 females with a median age of 64 years (range, 24-79 years). In the univariate analyses, the patients' age was an important factor in obtaining a favorable outcome $(P=0.041)$ (Table 1$)$. In addition, a cutoff age of 64 years was selected on the basis of the highest sensitivity (0.909) and specificity (0.606), as well as the area under the curve (0.755), generated from the ROC curves (Fig. 3). The overall 
Table 1 Patients' baseline characteristics

\begin{tabular}{|c|c|c|c|}
\hline \multirow{2}{*}{ Variable } & \multicolumn{2}{|c|}{ Functional outcomes at the end of follow-up } & \multirow{2}{*}{$P$ value } \\
\hline & Unfavorable (mRS >2) & Favorable (mRS $\leq 2)$ & \\
\hline Patients & $33(75)$ & $11(25)$ & \\
\hline Age (years) & $63[24-79]$ & $55[43-71]$ & 0.041 \\
\hline Male & $12(36)$ & $7(64)$ & 0.154 \\
\hline Initial systolic pressure $<180 \mathrm{mmHg}$ & $16(59)$ & $9(82)$ & 0.081 \\
\hline \multicolumn{4}{|l|}{ History } \\
\hline Hypertension & $14(42)$ & $6(55)$ & 0.509 \\
\hline Diabetes mellitus & $3(9)$ & $0(0)$ & 0.561 \\
\hline Dyslipidemia & $3(9)$ & $1(9)$ & 0.999 \\
\hline \multicolumn{4}{|l|}{ Aneurysm } \\
\hline Anterior circle of Willis & $29(88)$ & $8(73)$ & 0.341 \\
\hline Size $<10 \mathrm{~mm}^{*}$ & $21(66)$ & $8(89)$ & 0.240 \\
\hline \multicolumn{4}{|l|}{ Treatment } \\
\hline Operation within $\leq 24$ hours & $30(91)$ & $9(82)$ & 0.586 \\
\hline Surgical clipping for aneurysm & $31(94)$ & $9(82)$ & 0.256 \\
\hline Use of decompressive craniectomy & $17(52)$ & $7(64)$ & 0.728 \\
\hline Ventriculo-peritoneal shunt & $17(52)$ & $6(55)$ & 0.999 \\
\hline Intracerebral hematoma & $18(55)$ & $4(36)$ & 0.488 \\
\hline Acute hydrocepharus & $14(42)$ & $3(27)$ & 0.486 \\
\hline Ischemia by cerebrovascular spasm & $14(42)$ & $1(9)$ & 0.067 \\
\hline Length of hospital stay (days) & $59[4-275]$ & $75[24-211]$ & 0.377 \\
\hline
\end{tabular}

Data are presented as number (percentage) or median [range].

mRS: modified Rankin scale

*Except for three cases (dissecting aneurysms).

duration between onset and aneurysm repair was less than $24 \mathrm{~h}$ in $39(88.6 \%)$ patients, $24-48$ hours in $3(6.8 \%)$ patients, and $48-72$ hours in $2(4.5 \%)$ patients. The most common comorbid disorder among all patients was arterial hypertension $(\mathrm{n}=$ $20 ; 45.5 \%)$, followed by dyslipidemia $(\mathrm{n}=4 ; 9.0 \%)$, and diabetes mellitus ( $\mathrm{n}=3 ; 6.8 \%$ ).

The median size of ruptured aneurysms was 8.4 $\mathrm{mm}$ (range, $1.8-20.0 \mathrm{~mm}$ ), and three $(6.8 \%)$ patients had a dissecting aneurysm. These were more commonly located in the anterior Circle of Willis $(\mathrm{n}=37 ; 84.0 \%)$ than in the posterior Circle, and the most common locations of aneurysm were the internal carotid artery-posterior communicating artery ( $\mathrm{n}=12 ; 27.3 \%$ ) and the anterior communicating artery $(\mathrm{n}=12 ; 27.3 \%)$. Aneurysm repair was performed by means of surgical clipping in 40 (90.9\%) patients and by coiling in $4(9.1 \%)$ patients. Decompressive craniectomy was performed in 23 $(52.3 \%)$ patients, cranioplasty in $12(27.3 \%)$ patients, and ventriculoperitoneal shunting in 23 (52.3\%) patients during their hospital stay. The median hospitalization duration among all patients was 56.0 days (range, 4-211 days). After aneurysm repair, 10 $(22.7 \%)$ patients died during their hospital stay, and $9(20.5 \%)$ patients who were severely disabled were transferred to another hospital for recuperation. The remaining $25(56.8 \%)$ patients were moved to rehabilitation hospitals.

Figure 4 shows the study outcomes from discharge to 3 years after hemorrhage. Although no patient had a favorable functional outcome at discharge, $11(25.0 \%)$ patients had a favorable functional outcome at the end of follow-up. The number of patients with a favorable functional outcome significantly increased during the first year $(P=0.012)$ and during the follow-up period $(P<0.001)$. In contrast, none of the patients who were transferred to recuperation hospitals had a favorable functional outcome (Fig. 1).

\section{Discussion}

Our single-center population of most severe cases of SAH showed potential for marked functional 


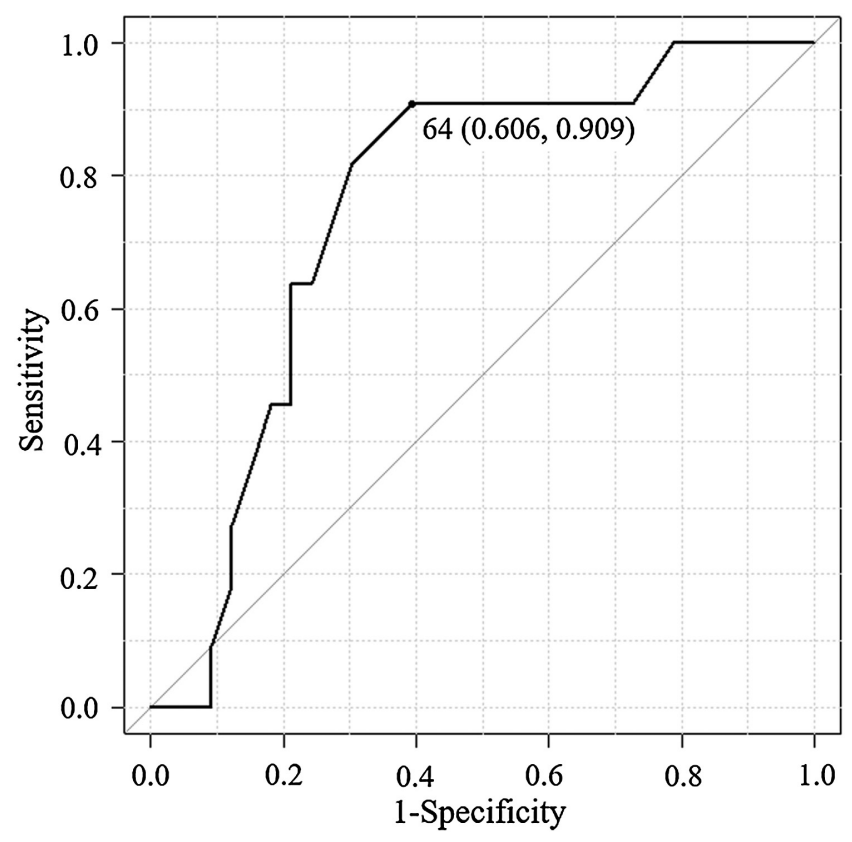

Fig. 3 Receiver operating characteristic curve plotted using the data for age and favorable outcome, and presented as the cutoff age (64 years) and the highest sensitivity $(0.909)$ and specificity $(0.606)$ values.

improvement with aggressive treatment. This improvement progressed over the course of at least 3 years, and finally, one-fourth of the patients had a favorable outcome at the end of the follow-up period.

Previous studies on outcomes in most severe SAH cases were diverse in their grading scales used to assess severity, as well as in the observation period ( 3 months to 3 years). We used the WFNS grading scale because of its objectivity and general usefulness. ${ }^{9)}$ To our knowledge, no previous study has had a follow-up period as long as that in the present study. Moreover, the proportion of favorable functional outcomes in the present study was at the upper limit of that reported previously, which ranged between $13 \%$ and $24 \% .{ }^{15-19)}$

Neurological grading and surgical indications are important indicators of outcomes in patients with grade V SAH. Determining the change in GCS score is difficult because patients with grade $\mathrm{V}$ SAH are often sedated. Furthermore, some patients may experience neurological recovery after resuscitation. Because of these reasons, it is preferable to assess the WFNS grade immediately before therapeutic interventions. In the present study, emergency physicians or neurosurgeons categorized the neurological status at the time of the treatment planning as shown in the flowchart of Fig. 2.

All patients who had a favorable outcome received sufficient rehabilitation as per their physical state, and by their choice of step-down facility. When a patient with grade V SAH is scheduled for discharge from our acute care hospital, we determine the next suitable medical facility for the patient, with consideration of their age, complications, progress status, and hope of recovery. Stroke rehabilitation is considered to lead to an improvement in the activities of daily life and the rate of return to the home. ${ }^{20)}$ In fact, guidelines from the American Heart Association and the Veterans Health Affairs suggest that all patients with stroke should receive rehabilitation that is as intensive as feasible. ${ }^{21,22)}$ The present study results show that a small proportion of patients had a favorable neurological outcome after a grade V SAH with active rehabilitation. This suggests that signs of recovery should be necessarily considered in the most severe cases of SAH to provide maximal possible opportunity for patients to undergo rehabilitation in the chronic phase to achieve a long-term favorable outcome.

Age may be another important factor associated with a favorable outcome as indicated by the longterm findings in the present study. ROC curve analysis suggests that an age of 64 years might be a criterion of favorable prognosis. Our results were consistent with those reported in previous studies with a relatively short observational period, which have shown an association between age and worse outcomes. ${ }^{7,18,23,24)}$

This study had several limitations. First, grading might be fraught with uncertainty. It is difficult to unequivocally standardize neurological status categorization as mentioned above. Therefore, the present study may have inadvertently included aneurysmal $\mathrm{SAH}$ with lower grades. However, grading immediately before sedation or other intensive care is the most suitable approach for assessment during initial treatment. If a patient requires sedation and intubation before diagnosis, the grading must refer to the GCS score allotted immediately before sedation, as this might be the last correctly evaluable consciousness level. Conversely, if a patient's vital signs are stable without resuscitation, the GCS score after diagnosis and immediately before starting intensive care should be considered. Nevertheless, it is important to assess a newer GCS score for grading. Although this topic remains controversial, compared with previous results, our indications might be more acceptable overall.

Second, although we reached consensus on the treatment strategy for grade $\mathrm{V} \mathrm{SAH}$, as shown in the strategy flowchart, the treatment decisions for each case were made at the discretion of the attending surgeon. Therefore, it is possible that the treatment plan was determined by subjective assessments, resulting in a selection bias. The standardization 


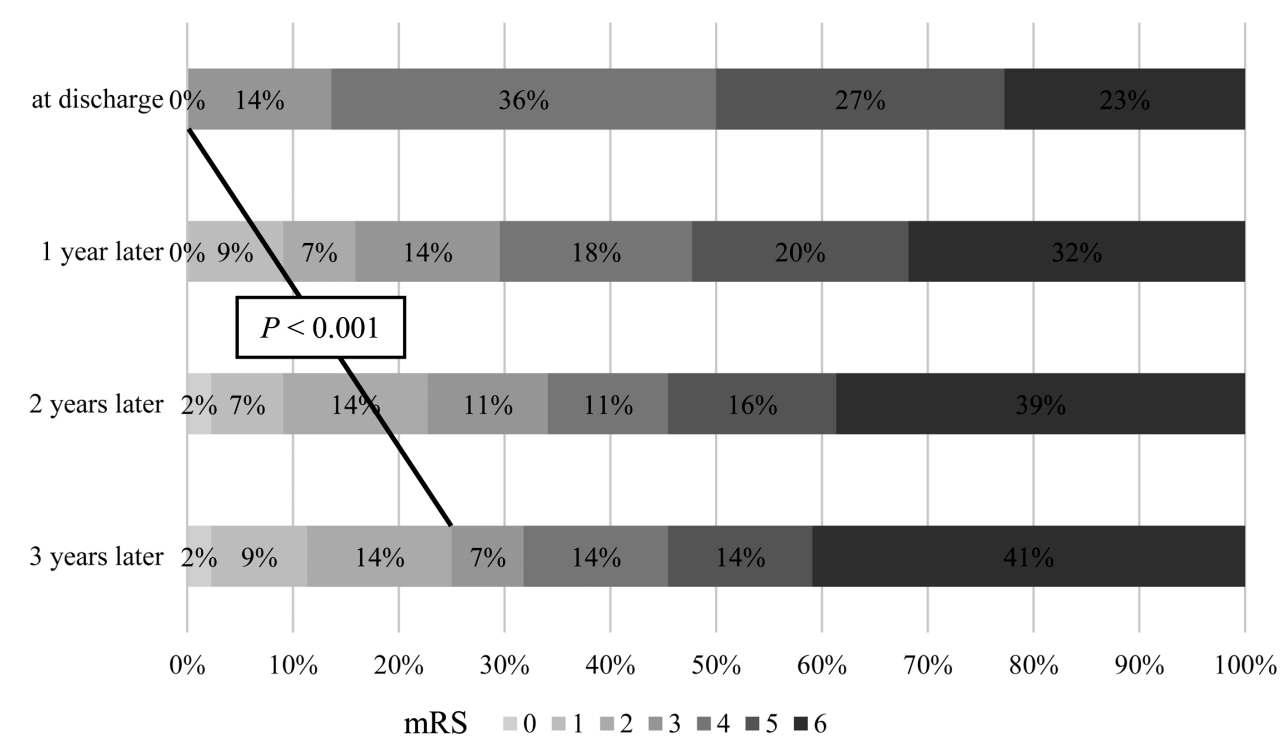

Fig. 4 The mRS scores of all patients from discharge to 3 years after subarachnoid hemorrhage. The number of patients with a favorable outcome increases markedly in the first year $(P=\mathbf{0 . 0 1 2})$. Analysis through the follow-up period reveals a significant increasing trend $(P<0.001)$. mRS: modified Rankin scale.

of intensive care, medications, and surgical procedures across facilities is desirable and remains a topic for future studies. However, this would be difficult because of diversity in the clinical course, patient background, personal distribution, and equipment.

Third, the precise period and program of rehabilitation remain unclear in our data, although this might be a meaningful prognostic factor. There is no useful protocol detailing a specific rehabilitation for the most severe cases of SAH. The importance of earlier rehabilitation in stroke patients is well known. We attempted to start rehabilitation as soon as possible, and to continue it until discharge, depending on the patients' condition (range of motion during exercises, swallowing training, speech therapy, higher brain function training, etc.). Because of limited medical resources, we had to classify patients into the following two groups at the end of acute care: a further rehabilitation group and a recuperation group. The distributions might depend on not only the patients' condition but also on other social factors (the number of remaining beds, family background, etc.). Therefore, an additional study is needed to describe the detailed relevance of such rehabilitation to the long-term outcomes, and to clarify the ideal rehabilitation for such patients. The issue of feasibility should also be considered.

Fourth, in the present study, only four patients $(9.3 \%)$ were treated by coiling. In recent years, the number of endovascular treatments for patients with severe $\mathrm{SAH}$ in the acute stage has increased rapidly. It has been reported that early $(<24 \mathrm{~h}$ after $\mathrm{SAH}$ ) coiling is associated with improved clinical outcomes at 6 months after the event. ${ }^{25)}$ Coiling is useful in patients with severe $\mathrm{SAH}$ because it is less invasive and less susceptible to cerebral swelling. In contrast, decompression and evacuation of hematoma are often required to control the intracranial pressure in patients with severe SAH. The optimal treatment procedure for ruptured aneurysms remains controversial, ${ }^{26)}$ and a large-scale comparative study of clipping and coiling with a large number of severe SAH cases and a long-term follow-up is needed.

In conclusion, the present study demonstrated that the functional outcomes of patients with WFNS grade V SAH improve over a period of 3 years, with one-fourth of the patients achieving a favorable functional outcome after aggressive treatment. This was based on the indications of initial vital signs, brainstem function, and aneurysm repair in the acute stage. A young age at SAH onset and efficient rehabilitation might have an impact on clinical practice, and these factors might play a role in improving clinical outcomes and prognosis. It is important to take the long-term outcome and prudent indications into consideration when deciding on a treatment strategy. Larger studies are required to adequately assess the long-term functional outcomes and other multi-faceted prognoses. 


\section{Conflicts of Interest}

All authors declare that they have no conflict of interest.

\section{References}

1) Connolly ES, Rabinstein AA, Carhuapoma JR, et al.; American Heart Association Stroke Council; Council on Cardiovascular Radiology and Intervention; Council on Cardiovascular Nursing; Council on Cardiovascular Surgery and Anesthesia; Council on Clinical Cardiology: Guidelines for the management of aneurysmal subarachnoid hemorrhage: a guideline for healthcare professionals from the American Heart Association/American Stroke Association. Stroke 43: 1711-1737, 2012

2) van Heuven AW, Dorhout Mees SM, Algra A, Rinkel GJ: Validation of a prognostic subarachnoid hemorrhage grading scale derived directly from the Glasgow Coma Scale. Stroke 39: 1347-1348, 2008

3) Ohkuma H, Tsurutani H, Suzuki S: Incidence and significance of early aneurysmal rebleeding before neurosurgical or neurological management. Stroke 32: 1176-1180, 2001

4) Naidech AM, Janjua N, Kreiter KT, et al.: Predictors and impact of aneurysm rebleeding after subarachnoid hemorrhage. Arch Neurol 62: 410-416, 2005

5) Ishihara H, Suzuki M: Japanese Guidelines for the Management of Stroke 2015: overview of the chapter on Subarachnoid Hemorrhage. Nihon Rinsho 74: 677-680, 2016

6) Suzuki M, Otawara Y, Doi M, Ogasawara K, Ogawa A: Neurological grades of patients with poor-grade subarachnoid hemorrhage improve after short-term pretreatment. Neurosurgery, 47:1098-1104; discussion 1104-1105, 2000

7) Laidlaw JD, Siu KH: Poor-grade aneurysmal subarachnoid hemorrhage: outcome after treatment with urgent surgery. Neurosurgery 53: 1275-1280; discussion 1280-1282, 2003

8) Le Roux PD, Dailey AT, Newell DW, Grady MS, Winn HR: Emergent aneurysm clipping without angiography in the moribund patient with intracerebral hemorrhage: the use of infusion computed tomography scans. Neurosurgery 33: 189-197; discussion 197, 1993

9) Report of World Federation of Neurological Surgeons Committee on a Universal Subarachnoid Hemorrhage Grading Scale. J Neurosurg 68: 985-986, 1988

10) Mitsuma W, Ito M, Kodama M, et al.: Clinical and cardiac features of patients with subarachnoid haemorrhage presenting with out-of-hospital cardiac arrest. Resuscitation 82: 1294-1297, 2011

11) Ogilvy CS, Carter BS: A proposed comprehensive grading system to predict outcome for surgical management of intracranial aneurysms. Neurosurgery 42: 959-968; discussion 968-970, 1998

12) van Gijn J, Hijdra A, Wijdicks EF, Vermeulen M, van Crevel H: Acute hydrocephalus after aneurysmal subarachnoid hemorrhage. J Neurosurg 63: 355-362, 1985
13) de Haan R, Limburg M, Bossuyt P, van der Meulen J, Aaronson N: The clinical meaning of Rankin 'handicap' grades after stroke. Stroke 26: 2027-2030, 1995

14) Kanda Y: Investigation of the freely available easy-touse software 'EZR' for medical statistics. Bone Marrow Transplant 48: 452-458, 2013

15) Schwartz C, Pfefferkorn T, Ebrahimi C, et al.: Long-term neurological outcome and quality of life after world federation of neurosurgical societies grades IV and V aneurysmal subarachnoid hemorrhage in an interdisciplinary treatment concept. Neurosurgery 80: 967-974, 2017

16) Le Roux PD, Elliott JP, Newell DW, Grady MS, Winn HR: Predicting outcome in poor-grade patients with subarachnoid hemorrhage: a retrospective review of 159 aggressively managed cases. J Neurosurg 85: 39-49, 1996

17) Seifert V, Trost HA, Stolke D: Management morbidity and mortality in grade IV and V patients with aneurysmal subarachnoid haemorrhage. Acta Neurochir (Wien) 103: 5-10, 1990

18) van den Berg R, Foumani M, Schröder RD, et al.: Predictors of outcome in World Federation of Neurologic Surgeons grade V aneurysmal subarachnoid hemorrhage patients. Crit Care Med 39: 2722-2727, 2011

19) Chiang VL, Claus EB, Awad IA: Toward more rational prediction of outcome in patients with high-grade subarachnoid hemorrhage. Neurosurgery 46: 28-35; discussion 35-36, 2000

20) Ottenbacher KJ, Jannell S: The results of clinical trials in stroke rehabilitation research. Arch Neurol 50: 37-44, 1993

21) Duncan PW, Zorowitz R, Bates B, et al.: Management of adult stroke rehabilitation care: a clinical practice guideline. Stroke 36: e100-143, 2005

22) Bates B, Choi JY, Duncan PW, et al.; US Department of Defense; Department of Veterans Affairs: Veterans affairs/department of defense clinical practice guideline for the management of adult stroke rehabilitation care: executive summary. Stroke 36: 2049-2056, 2005

23) Lanzino G, Kassell NF, Germanson TP, et al.: Age and outcome after aneurysmal subarachnoid hemorrhage: why do older patients fare worse? J Neurosurg 85: 410-418, 1996

24) Mocco J, Ransom ER, Komotar RJ, et al.: Preoperative prediction of long-term outcome in poor-grade aneurysmal subarachnoid hemorrhage. Neurosurgery 59: 529-538; discussion 529-538, 2006

25) Luo YC, Shen CS, Mao JL, Liang CY, Zhang Q, He ZJ: Ultra-early versus delayed coil treatment for ruptured poor-grade aneurysm. Neuroradiology 57: 205-210, 2015

26) Inamasu J, Nakamura $Y$, Saito R, Kuroshima $Y$, Mayanagi K, Ichikizaki K: Endovascular treatment for poorest-grade subarachnoid hemorrhage in the acute stage: has the outcome been improved? Neurosurgery 50: 1199-1205; discussion 205-206, 2002

Address reprint requests to: Kenichi Ariyada, MD, Department of Neurosurgery, Tokyo Metropolitan Bokutoh Hospital, 4-23-15 Kotobashi, Sumida-ku, Tokyo 130-8575, Japan e-mail: arikyaken@yahoo.co.jp 